\title{
Treatment outcomes after endopyelotomy performed with or without simultaneous nephroli- thotomy: 10-year experience
}

Berkman DS, Landman J, Gupta M

Department of Urology, Columbia University, College of Physicians and Surgeons, New York, New York, USA

J Endourol. 2009; 23: 1409-13

Introduction: The incidence of renal calculi associated with ureteropelvic junction obstruction (UPJO) has been reported to be as high as $20 \%$. Although it has been suggested that simultaneous stone removal be performed with endopyelotomy (EP) for patients with UPJO, no crossing vessel, and renal calculi, there are no large series to date reporting in a rigorous fashion the success rate for resolution of the UPJO. This study intends to determine if stone extraction performed with EP increases failure rate.

Materials and Methods: A retrospective review was performed for all patients who underwent EP by a single surgeon between August 1996 and November 2006. One hundred forty-six procedures for UPJO were done in 139 patients. Success rate was compared in 105 cases (72\%) of isolated UPJO and 41 (28\%) cases with UPJO and ipsilateral calculi. Determination of overall success required subjective improvement and objective data based on intravenous pyelogram, computed tomography, and/or nuclear scintigraphy.

Results: Overall success rate was $71 \%$ in patients undergoing EP only and $90 \%$ in 41 patients who had simultaneous nephrolithotomy $(\mathrm{p}=0.04)$. The odds ratio of failure after EP was 2.9 for severe/massive preoperative hydronephrosis. When considered alone and after adjusting for the severity of preoperative hydronephrosis and/or renal function, simultaneous nephrolithotomy did not increase the EP failure rate.

Conclusion: This study demonstrates that UPJO resolves at an equal rate after EP performed with or without ipsilateral renal calculi. Patients with UPJO and renal calculi should undergo stone extraction and EP in the same setting with the expectation of excellent results.

\section{Editorial Comment}

Traditionally a staged approach (stone extraction; re-evaluation; endopyelotomy) has been recommended for stones at the ureteropelvic junction as it is difficult to establish the presence of a primary UPJ obstruction versus the possibility of secondary obstruction due to edema that will resolve following stone extraction. Indeed, such patients were excluded from analysis in this study. Concerns are raised with regards to the possibility of fragment migration through the endopyelotomy incision if the two procedures are performed simultaneously. Unfortunately, the authors did not compare success rates for those undergoing intracorporeal lithotripsy to those undergoing intact stone removal, nor do they correlate success with stone size. Similarly, the authors do not report the presence of residual fragments or fragments in the retroperitoneum on postoperative imaging. The study could be criticized due to the lack of standardization in preoperative and postoperative imaging. The authors do not discuss their preoperative imaging to assess for crossing vessels, and whether the identification of crossing vessels impacted their treatment algorithm.

Dr. Manoj Monga

Professor, Department of Urology University of Minnesota Edina, Minnesota, USA E-mail:endourol@yahoo.com 